\title{
A Language-Based Approach to Protocol Implementation
}

\author{
Mark B. Abbott and Larry L. Peterson* \\ Department of Computer Science \\ University of Arizona \\ Tucson, AZ 85721
}

\begin{abstract}
Morpheus is special-purpose programming language that faciltates the efficient implementation of communication protocols. Protocols are divided into three categories, called shapes, so that they can inherit code and data structures based on their category; the programmer implements a particular protocol by refining the inherited structure. Morpheus optimization techniques reduce per-layer overhead on time-critical operations to a few assembler instructions even though the protocol stack is not determined untıl runtime. This supports divide-and-conquer simplification of the programming task by minimizing the penalty for decomposing complex protocols into combinations of simpler protocols.
\end{abstract}

\section{Introduction}

Network software is difficult to design and implement. As with any distributed concurrent program with complex functionality. correctness is difficult to achieve. Thus situation is exacerbated by the additional requirement of high performance. This paper introduces a new approach to the network software problemusing a programming language designed specifically for high performance protocol implementations. We have designed such a language, called Morpheus.

The foremost advantage of a programming language is that it is an ideal vehicle for both imposing and benefiting from constrants. In other words, a language provides both the means to restrict the design choices available to the programmer, and the medium in which to realize the advantages due to the narrower design domain. The constraints imposed by Morpheus take the form of strategies and techniques carefully selected

\footnotetext{
${ }^{*}$ This research supported in part by DARPA Contract DABT63-91C- -0030
}

Permission to copy without fee all or part of this material is granted provided that the copies are not made or distributed for direct commercial advantage, the ACM copyright notice and the title of the publication and its date appear, and notice is given that copying is by permission of the Association for Computing Machinery. To copy otherwise, or to republish, requires a fee and/or specific permission.

COMM'92-8/92/MD,USA

(6) 1992 ACM 0-89791-526-7/92/0008/0027 ..\$1.50 from among those exposed by collective experience with networks $[1,10,17]$. plus a novel constraint (shape) introduced in this paper.

Stated another way, there are two motivations for the constraints imposed by Morpheus. Frrst, they enforce a good design discipline. It has been argued that the development of a new engineering discipline often happens in two phases [8 ]. In the first phase, the capabilities of tools are expanded to cope with the growing set of problems. In the second phase, tools Impose a carefully selected set of constrants on the engineer in order to enforce a design discipline based on accumulated experience. Morpheus is a tool of the second phase: it is a special purpose programming language that provides an explıcit model for thinking about and concisely expressing protocols in accordance with a design discipline.

The second motivation is that it makes possible a more powerful tool. In effect, the more the user is constrained, the more the tool knows about what the user wants to do. For example, protocols written in Morpheus can be compiled into more efficient object code than those written in general purpose languages because a Morpheus compiler has more domain knowledge available to apply to low level optimization.

Morpheus adopts an object-oriented programming style. In Morpheus, the fundamental protocol abstractions are represented as objects. The Morpheus programmer implements a particular protocol by refining the code and data structures inherited from pre-defined base classes. The shape constraint serves to maximize the amount of code and data structures that can be ınherited.

Morpheus optimizations reduce per-layer overhead on tımecritical operations to a few assembler instructions, even though the protocol graph is not determined untıl runtıme. This supports divide-and-conquer simplification of the programming task by minimizing the penalty for decomposing complex protocols into combinations of simpler protocols.

Morpheus is more than a protocol model added to an existing language. Morpheus performs optimizations that existing languages cannot because they lack Morpheus' built-in knowledge of the common patterns of use of the elements of the model. It also manages the machine-dependent aspects-alignment and byte order-of message header manipulation. Although we have not yet exploited it. Morpheus might also be used to hide the granularity of concurrency on a multiprocessor.

This paper makes two important contributions. First, it describes the Morpheus programming model, most notably the shape constraint. Second, it discusses the optimization tech- 
nıques available to the Morpheus compiler. We document the effectiveness of these techniques with hand-coded optimizations in assembler language for the MIPS R3000 architecture [11].

\section{Background}

Morpheus was not designed in a vacuum. It reflects our understanding of network software based on experience bulding tools to support the rapid implementation of efficient protocols. This section identifies related work that has influenced Morpheus and discusses the biases in its design.

\subsection{Related Work}

Generally speaking, there are two common approaches to implementing network protocols. At one extreme, the protocol is written in a general purpose language, subject only to the constraints imposed by the operating system and architectural environment in which the protocol will be used. Although protocols implemented in this way are generally efficient, the programming task can be exceptionally difficult, depending on the extent to which the host operating system is designed to accommodate network protocols. In the best case, the operating system provides explicit support for implementing protocols. For example, the $x$-kernel provides a uniform protocol interface and a protocol support library [10].

At the other extreme, network software is automatically derived from a protocol specification expressed using a Formal Description Technique (FDT) such as Estelle, LOTOS, or SDL [16]. In its current state, however, this technology has not lived up to its promise. Instead of expressing a protocol in purely abstract terms, protocols are specified in relatively "implementation-oriented" FDTs, and the software generated is generally in the form of a skeleton which must be completed with programmer code.

This paper introduces a language-based approach to protocol implementation that lies between these two extremes. Our language-based approach has several advantages. First, it affords the opportunity to perform low level optimizations specific to protocol implementations. Second, code and data structures that are routine parts of protocols can be automatically provided. just as procedure prologue and epilogue code is automatically generated by general purpose languages. Third, a language can present a seamless model of protocols-no underpinnings are visible below the single level of abstraction.

Our approach is not independent of the two alternatives; it is related to the OS-based and the FDT-based approaches as follows.

We were led to this approach by our experience with the $x$ kernel, which takes the OS-based approach. Specifically, we realized there were several ways in which we wanted to extend the $i$-kernel that could best be accomplished by embedding .1 kernel-style protocol abstractions in a language. Morpheus is the result: it offers a more convenient way to express protocols by replacing the "bollerplate" found in $x$-kernel protocols with automatically generated code, it implements the operatıng system aspects of the $x$-kernel in the language's runtime system, and it takes advantage of compile-time optimizations not avarlable in a general purpose programming language. A considerable additional advantage which we have not pursued is the potential for a Morpheus debugger that would share Morpheus' knowledge of the structure and behavior of protocols.

Morpheus is like FDTs in that it strives to present a higher level of abstraction to the programmer/specifier. However, Morpheus' level of abstraction is not as high as that of FDTs; programmers still write in a familiar, imperative programming style. Furthermore, Morpheus is far less general, imposing constraints that eliminate design choices. But it is just this combination that makes it possible to present the programmer with the right design options and a relatively high level of abstraction from which complete, high-performance implementations can still be generated with current software technology.

\subsection{Architectural Biases}

One of the liberties we have taken with this research is to imagine what network software could look like if not constrained by today's standardized protocols. Current protocols often include artifacts that are not fundamental to network communication and that interfere with innovations. While we are interested in providing the same communication services (semantics) as are available in today's networks, we do so without regard for the exact form (syntax) of today's protocols.

In particular, Morpheus supports a dynamic network architecture, such as the one described in [12]. This architecture has two key characteristics:

- There are many, very simple protocols.

- Protocols are selected and combined at runtıme.

These characteristics have ramifications that are apparent in the design of Morpheus. Consider the following two biases.

First, Morpheus has a bias towards composing network software from the simplest possible protocols, going so far as to require a certain degree of simplicity. This has decisıve advantages when compared to using shorter stacks of more complex protocols. The primary advantage is that of divide-and-conquer: a complex protocol is harder to develop, verify, implement, and maintain than an equivalent collection of simple protocols. A second advantage is that of reuse. Reuse of protocol implementatıons requires not only that interfaces are syntactically compatible, but also that the composition of semantics is useful. Large complex protocols are unlikely to implement the precise combination of functions that is appropriate in another context. A third advantage of simpler protocols is the increased potential (exploited by Morpheus) for automatically generating parts of an implementation.

Additional advantages of simple protocols emerge when they can be selected and combined into a protocol stack, or more generally, a protocol graph. For one thing, a communication service user can configure exactly the communication service (i.e. protocol graph) needed, instead of sharıng a statically determined graph. Also, the development of new protocols is easier because binding decisions are delayed. Finally, it is easier to adapt to changes in the underlying technology; i.e., substitute new protocols that are better suited for the new technology.

Morpheus' second bias is against logical multiplexing - - the combining by a protocol of multiple higher-level data streams 
into a single lower-level data stream. This reflects the growing recognition of the penalties for logical multiplexing $[6,15]$ :

- When streams are merged, they can't be distinguished for purposes of quality of service.

- Multiplexing and demultiplexing at multıple layers hurts performance by duplicating effort.

- Multiplexing is a barrier to the propagation of flow and congestion control information between protocol layers.

Morpheus does not assume that every protocol multiplexes; in fact. Morpheus relegates the multiplexing protocols to a special category of protocol. Furthermore, Morpheus' inciusion of an interface for propagating flow control information between layers is predicated on the assumption that relatively few layers multiplex.

\section{Language Abstractions}

There are two main design goals behind Morpheus" language abstractions. First, the fundamental network abstractions such as messages and connections should be an integral part of the language. Second, Morpheus should automatically supply the predictable code and data structures appropriate for a given protocol. This second goal grew out of our experiences with the $x$-kernel. As is often the case in writing software, one programs an $x$-kernel protocol by first copying the code from a similar protocol to use as a template, and then editing that code to obtain the desired protocol. This approach derives its benefit from the fact that there are routıne tasks, such as manipulatıng headers and demultiplexing, that are common to many protocols. Morpheus has the goal of performing the equivalent task automatically.

Note that automatıcally supplying code constrains the programmer in that it preempts design choices, reducing certain design options to a single "option" for which Morpheus can supply the code. The obvious benefit is that the programmer doesn't have to write that code. The less obvious benefit is that in these cases, the programmer can't make a bad design chotce.

\subsection{Object-Based Design}

Morpheus represents the fundamental protocol abstractions as objects. Morpheus pre-defines a collection of base classes, and the programmer implements a protocol by refining these base classes to produce subclasses that are approprate to the specific protocol, as illustrated in Figure 1. An instance of a protocol is made up of objects which are instances of the subclasses specific to that protocol. Representing protocol abstractions this way not only achieves our goal of making the fundamental abstractions explicit in the language, but it also supports our second goal by providing a language-level mechanısm-inheritance-for supplying pre-defined code and data structures.

Morpheus' model of protocols divides them into categories, called shapes, based on therr functionality. Shapes are a particularly novel aspect of Morpheus. By constraining protocols to conform to these shapes, Morpheus gains additıonal information about each protocol which it uses to supply more code than would be possible for arbitrary protocols.

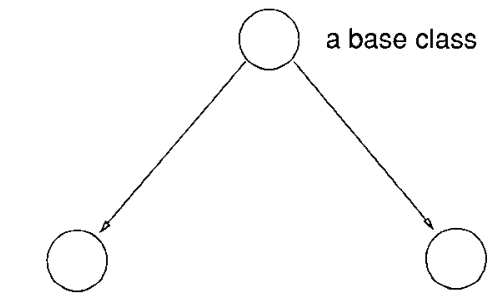

a subclass

a subclass used in protocol A used in protocol B

Figure 1: Protocols as Refinements

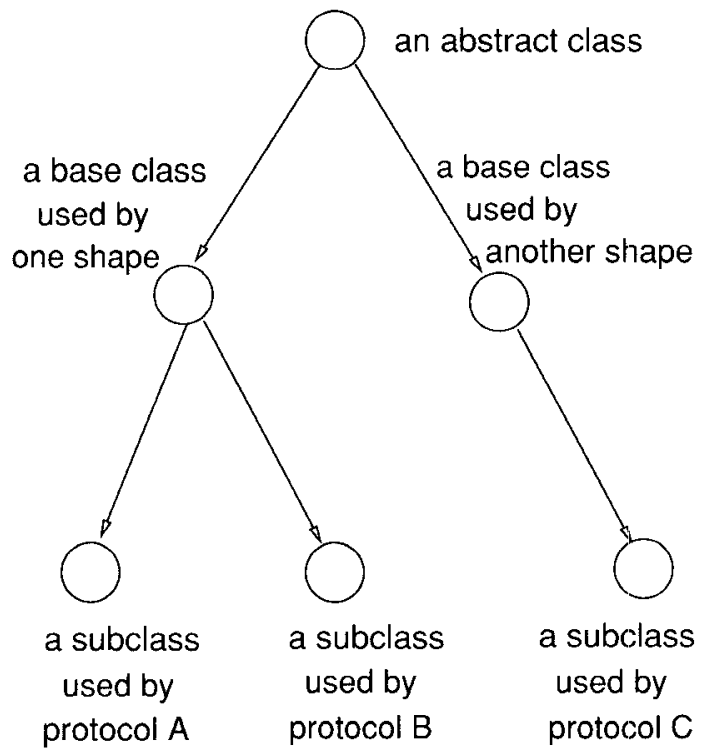

Figure 2: Classes and Shapes

In order to supply shape-based code via the mechanism of inheritance, the shape dimension must be integrated with the class hierarchy. Figure 2 schematically depicts the relationshıp between classes and shapes. The abstract classes at the top of the hierarchy specify interfaces used by all protocols. Each base class at the middle level of the herarchy adds code and data structures appropriate for all protocols of the corresponding shape. Each subclass at the bottom of the herarchy adds the remainıng code and data structures to complete the implementation of a specific protocol.

Object-oriented programming is well suited for representıng our protocol abstractions. One characteristic of our model of protocols is that it partitions state information such that each action operates on a specific body of state information. Objectonented programming fosters this way of thinkıng by packaging data together with related procedures.

Another benefit is notational economy. Procedures implementing operations on an object (the selt object) can refer to the state variables of that object directly, without explicit reference 
to the object. This benefit is multiplied in Morpheus because the ability to directly refer to state variables is extended to those objects of which the self object is a component. In other words. some Morpheus objects are contained in other objects, in which case they can refer to the state variables of those containing objects.

A final benefit is that object-oriented programming provides inheritance as a language-level discipline for automatically supplying behavior and data structures. Alternative techniques that are outside the language- - such as macros or library routinesmight be workable, but their benefit would be offset by the burden of working with mechanisms outside the language.

It should be made clear that Morpheus is object-oriented only with respect to the built-in protocol abstractions; the Morpheus programmer cannot define completely new classes. Also note that Morpheus' benefits could not be duplicated by adding predefined classes to a general object-oriented language such as $\mathrm{C}++[14]$ since it would lack the knowledge of common patterns of protocol operation invocation that Morpheus exploits to optimize. Also, a general purpose language could not manage alignment and byte order considerations for message headers. Finally, certain syntactic niceties would have to be sacrificed.

\subsection{Base Classes}

Morpheus defines five programmer-refined base classes, corresponding to the fundamental elements of Morpheus' model of protocols. The Morpheus programmer implements a protocol by refining the base classes, thereby deriving subclasses that are specific to the protocol. A subclass is derived from a base class by adding new state information (declaring additional in stance variables) and by modifying and extending the base class behavior (defining additional procedure code that augments or overrides the base class procedures).

The five base classes-Protocol, OverSap, UnderSap, OverSession, and UnderSession-are schematically depicted in Figure 3. Note that some objects are nested in others; a Protocol object includes as components some OverSap and UnderSap objects, OverSaps in turn have OverSessions as components, and likewise UnderSaps include UnderSessions as components.

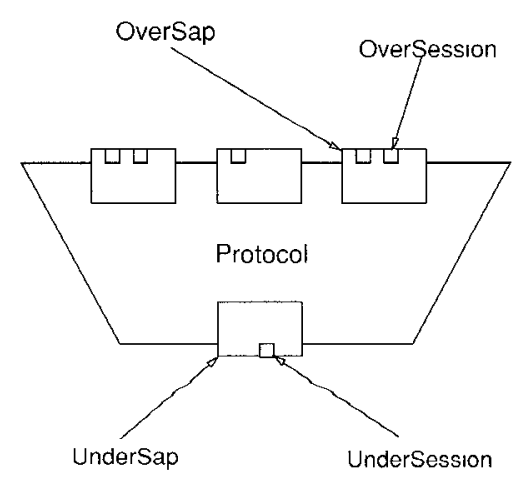

Figure 3: Base Classes

There are two kinds of operations associated with objects of these classes: e.ternal and internal. External operations are operations that can be invoked in Morpheus code. External operations are implemented by infrastructure provided by Morpheus: this infrastructure invokes the corresponding internal operations. It is the internal operations that the protocol programmer must Implement. There are several reasons for the distinction between internal and external operations. First, some external operations translate into a combination of several internal operations. Second, the infrastructure in some cases performs significant work itself. Finally, objects cannot directly invoke internal operations on objects belonging to other protocols because this would entail the ability to refer to those objects, violating a valuable protocol-granularity encapsulation.

The object operations are summarized in Table 1. For purposes of research, we have selected a minımal functional set of protocol operations. A practical system would require additional operations.

A Protocol object represents a protocol entity: an active instance of a particular implementation of a protocol specification. Each instantiation of a given protocol in a host's protocol graph is a distinct protocol entity.

OverSap and UnderSap objects represent Service Access Points, or SAPS. A SAP is a communication service interface with an address that uniquely identifies it. The interface between one particular protocol entity and another, higher level protocol entity as identıfied by a given address is a SAP. The communication service user side of a SAP is represented by a UnderSap object, which is part of the user Protocol object, while the service provider side is represented by an OverSap object, which is part of the provider Protocol (SAP objects are named from the point of view of the Protocol of which they are components; see Figure 3). Figure 4 illustrates a complete SAP consistıng of a matched UnderSap-OverSap pair.

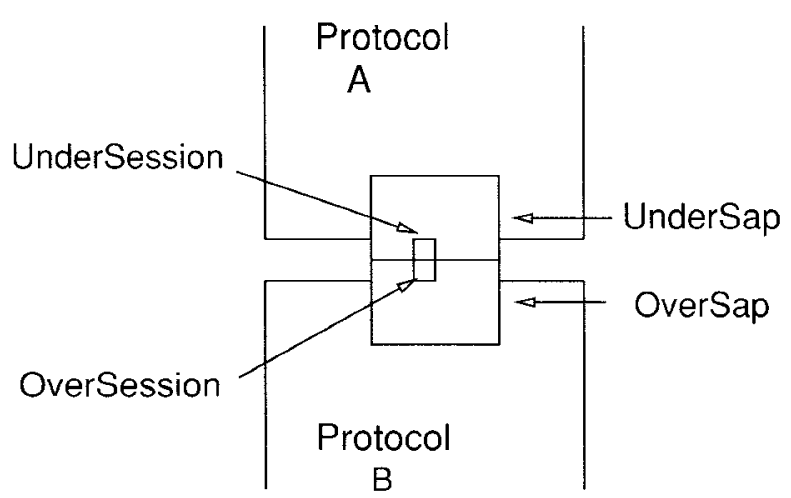

Figure 4: Sap and Session objects

OverSession and UnderSession objects represent sessions. A session is an endpoint of a data stream or abstract communication channel (not necessarily a connection) between two specific SAPs. Hence, there are two addresses associated with a session: the address of the SAP at "this end," and the address of the SAP at "the other end." A session is the interface between the protocol entity that uses the communication channel and the protocol 


\begin{tabular}{|r|r|}
\hline EXTERNALLY INVOKED OPERATIONS & CORRESPONDING INTERNAL OPERATIONS \\
\hline createProtl(underSaps) & $\begin{array}{r}\text { protl.Int( underSaps) } \\
\text { protl.addOverSap(overSap) }\end{array}$ \\
\hline underSap.getLocalAddr() & overSap.getLocalAddr() \\
\hline underSap.createUnderSessn(addr) & $\begin{array}{r}\text { overSessn.initOverSessn(addr) } \\
\text { underSessn.InitUnderSessn(addr) }\end{array}$ \\
\hline overSap.createOverSessn(addr) & $\begin{array}{r}\text { overSessn.initOverSessn(addr) } \\
\text { underSessn.initUnderSessn(addr) }\end{array}$ \\
\hline underSessn.send(msg) & overSessn.send(msg) \\
\hline overSessn.deliver(msg) & underSessn.deliver(msg) \\
\hline overSessn.grantSends(number) & underSessn.grantSends(number) \\
\hline underSessn.grantDelivers(number) & overSessn.grantDelivers(number) \\
\hline
\end{tabular}

Table 1: Object Operations

entity that provides the channel. The user side of a session is represented by an UnderSession object, and the provider side is represented by an OverSession object. Figure 5 illustrates UnderSession-OverSession pairs and data streams.

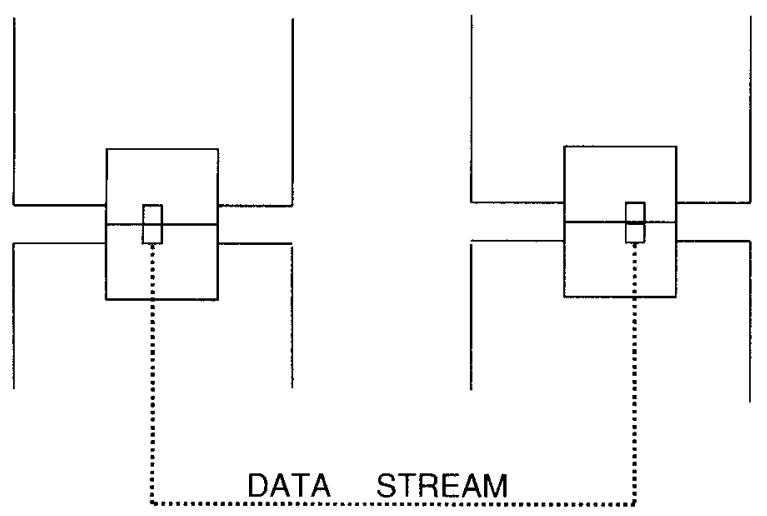

Figure 5: Sessions and Data Streams

UnderSession objects have a deliver(message) operation (messages are delivered asynchronously, rather than being received) and a grantSends(numberOfMessages) operation used to convey flow control information. OverSession objects have the analogous operations send(message) and grantDelivers(numberOfMessages).

Note that while the five base classes just defined must be refined by the programmer to derive specific protocols. Morpheus provides three additional classes that are not refined by the programmer: Message, Map (a kind of hash table) and Event (a schedulable event). These classes represent utilities frequently used by protocols. They play a role similar to library routines in other languages, but are built into the language as object classes. The programmer uses objects from these utility classes to help write the five protocol-specific classes.

\subsection{Shapes}

Morpheus constrains protocols to conform to one of three shapes: multiplexor, router, or norker. The purpose of this powerful constraint is to maximize the information that the Morpheus compiler can use to automatically supply code and data structures. This constraint has been carefully selected so as to avoid restricting the range of protocol functionality that can be implemented. Morpheus shapes result from partitioning protocol functionality on the basis of addressing. What we mean by "addressing" will be made clear as the three shapes are defined below. This particular partition provides critical information about the structure of a protocol-permitting the compiler to supply much more code than would otherwise be possiblewhile still supporting the full range of protocol functionality. Figure 6 schematically depicts the three protocol shapes.
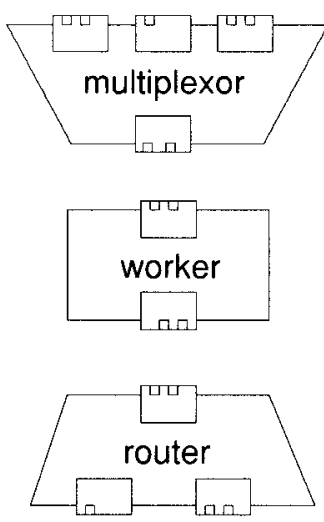

Figure 6: Three Shapes

Multiplexor protocols multiplex and demultiplex. They operate on the multiplexing keys associated with different users, but are ignorant of host addresses. They may use quality of service (QOS) information associated with user SAPs in performing multiplexing. A multiplexor protocol provides a variable number of OverSaps (since it may multiplex channels from many users), but uses just one UnderSap. Multiplexors can use flow control information regarding sending messages to schedule outgoing messages, but cannot enforce flow control on delivery of messages (if needed, it must be implemented in a separate protocol). 
Router protocols deal with host addresses. They interpret host addresses, but never see multiplexing keys. A router protocol provides just one OverSap and uses some fixed number of UnderSaps. Note that Morpheus routers are more general than is usually suggested by the term "router" (e.g. IP), in that we include choosing between different paths within the protocol graph. More precisely, a router is any protocol that must decide at runtıme which lower level data stream (UnderSession) to use for a given higher level data stream (OverSession).

Worker protocols do what might be described as "the real work" such as error detection, buffering for retransmission, and detecting lost, reordered, or duplicated messages. In particular, any manipulations of message data are performed by workers. They don't process host addresses, and they never see multiplexing keys. A worker protocol entity provides one OverSap and uses one UnderSap.

Without distinguishing protocol shapes, there is little code or state information that is common to all protocols. However, protocols of a given shape are similar enough that they can usefully inherit default behavior and state variables. For example, all multiplexors do the same thing when messages are delivered to them: demultıplex them. Hence, the deliver operation is completely specified for multiplexors. In contrast, little deliver behavior can be supplied to routers or workers because they may perform widely varying functions. For another example, each worker protocol has a single user, hence a single OverSap. A state variable representing that OverSap is automatically declared in the Protocol base class for workers. Multuplexors, which may have many OverSaps, do not have this state var1able. Instead, multiplexors automatıcally get two state variables which are Map objects for mapping from an incoming message to the appropriate OverSap.

The Morpheus program for a protocol begins by explicitly statıng the protocol's shape. This allows the Morpheus compiler to implicitly provide data structures and behavior based on the shape, thus relieving the programmer of the burden of designing and implementing them. Programmers augment the provided data structures with additional data structures, and augment or override the provided code with their own.

Note that although protocols that are functionally equivalent to protocols such as TCP and IP can be implemented in Morpheus, those specific protocols - as specified in their standardscannot be implemented in Morpheus. This is because they combine the functions of more than one shape in a single protocol. For example, IP performs multiplexing, routing, and fragmentation/reassembly. In Morpheus these functions would be implemented as three distinct protocols: a multiplexor, a router, and a worker, respectively.

It is not surprising that existing protocols violate the shape constraint. One reason is the belief, refuted in this paper and [12], that efficiency requires that there be very few layers in a protocol stack. This encourages large, complex protocols that comprise multiple functionalities. A second reason is that ex1sting protocols were designed before the current acknowledgement of the drawbacks of logical multiplexing $[6,15]$ : hence many existing protocols include logical multiplexing among their functions, even though they need not.

\subsection{Inheritance}

We now consider how code inherited from Morpheus shapes 1s integrated with a Morpheus program written for a specific protocol. The general problem of integrating superclass behav1or with subclass behavior is known as the method combination problem [9]. The Morpheus case is much simpler than the general case because a subclass in Morpheus inherits from a single superclass, the superclass does not inherit any behavior, the superclass is never mstantiated directly (it is in this sense an abstract class), and the programmer cannot define completely new classes.

Morpheus uses a generalization of the method combination technique used in Simula [5]. In Simula, the keyword inner is used in a superclass operation definition to indicate that subclass code for this operation should be executed (like a subroutıne or macro) at this point in the superclass code. Unlike most other object-oriented languages, this requires the programmer to structure code top-down-the superclass has to anticipate how it will be augmented by subclasses. A top-down structure is ideal for Morpheus since the superclasses are pre-defined by the system, and superclass behavior is integrated in a fixed way.

More concretely, Morpheus does the following. In the program for a protocol the programmer writes procedures for the object operations, naming each procedure with its operation name. This code is inserted into the base class code for the same operation. The use of procedures here is simply a syntactic convention; subclass code is combined with base class code at compile time, so there is no procedure call overhead. If a subclass doesn't need to augment the base class code for a given operation, it does not define the corresponding procedure. The procedures corresponding to some operations take parameters which differ from those of the operations. This is because the role of these parameters is to let the subclass procedure refer to context in the base class code. This is not as cluttered as it might sound because most context is implicit: the object on which the operation was invoked, and any objects of which that object is a component.

Morpheus has two features not supported by the basic mechanism just described. The first is the ability to overnde base class behavior. This permits base classes to offer default behavior even though that behavior might not always be desired. The second feature is the ability to intermix base class and subclass code at a finer granularity. Base class and subclass behavior do not always fall into the neat relationship required by inner. It is useful for some base class operations to include different blocks of subclass code, each at a different point. Extending the basic mechanism to support these features is easy because Morpheus doesn't require a general solution-the base classes are pre-defined so the instances of these features are fixed. The new code is again packaged as a procedure; the difference is that the name of the procedure is not the name of an operation. The procedure is named by a keyword corresponding to the appropriate overridable block of code or location where new code can be inserted. The keywords and their semantics are as easy to learn as the operations because, like the operations, they are few and correspond to meaningful units of behavior. 


\section{Example}

The code in Figure 7 is the Morpheus program for a worker protocol called "Sequencer." Sequencer"s function is to filter out any duplicate or out-of-order packets. Note that Sequencer does not guarantee that every message sent is delivered; that would be the function of another layer.

The reader should note the almost complete absence of any code or data structures that are not specific to Sequencer's function. In contrast, an implementation of Sequencer in a general purpose language would include data structure declarations and code for creating and assembling the component objects, connecting Sequencer to the adjacent layers, creating data streams, and pushing and popping message headers. Using Morpheus, these routine aspects of a worker protocol are all inherited. The complete Sequencer protocol can be succinctly defined because one need express only those design choices that are specific to Sequencer.

The identifiers underSession (used in send) and overSession (used in deliver) are examples of inherited state variables. Since a worker doesn't do any address translation, there is a one-toone correspondence between UnderSessions and OverSessions. Variable overSession gives the UnderSession's corresponding OverSession object, and variable underSession gives the OverSession's corresponding UnderSession object. Sequencer uses other state variables that are not explicit in Sequencer's Morpheus program because they are used exclusively by inherited code.

The initialization of the state variable overSession is an example of inherited behavior. InitUnderSession() is invoked when Sequencer opens a channel of the underlying communication service to initialize the UnderSession representing Sequencer's side of the interface to that channel. The inherited base class code for initUnderSession takes care of setting the UnderSession's overSession to the OverSession representing the corresponding Sequencer channel.

The keyword LittleEndian indicates the byte order with which the sequence number seqNum in the header is to be represented. The compiler uses this information to automatically generate the appropriate code for reading and writing header fields. This highlights an obvious advantage of a language designed exclusively for writing network protocols: the compiler can worry about network byte order and byte alignment.

Multiplexor protocols provide a more dramatic example of inheritance. The code in Figure 8 is the Morpheus program for a multiplexor protocol called "FCFS."

Compared to a worker protocol, more of a multiplexor is specified in the base classes because more is known about the function of a multiplexor. FCFS inherits specific algorithms and data structures for the basic multiplexing and demultiplexing tasks performed by every multiplexor.

The dimension along which multiplexors vary is the scheduling of outgoing messages. FCFS is the simplest useful multiplexor, schedulıng outgoing messages first-come-first-serve. More sophisticated multiplexors transmit messages when permitted by the flow and congestion control information conveyed via the grantSends operation, and transmit them in an order based on priority or quality of service (QOS) considerations.

Certainly, more complex protocols require more code than these examples. The essential point of the examples is not that they are short, but rather that they don't require expression of the "routine" code that is common to all protocols of a the corresponding shape, and this is equally true for more involved protocols.

\section{Performance Optimizations}

This section identifies some domain-specific optimizations available to a Morpheus compiler, and reports experimental results based on performing these optimizations by hand.

\subsection{General Strategy}

Morpheus optimization techniques are based on the common patterns of protocol execution. Consider the characteristics of the send operation; deliver behaves similarly. Send takes a message as its argument; hence there are in effect two arguments, the message and the OverSession object. The typical send does some computation, accessing the object for state and other information, and using the built-in utilities to manipulate messages, hash tables, and timers; prepends a header to the message; and ends by passing the message to the next lower layer via the send operation of another OverSession. This is repeated as the message passes through "many" layers. Morpheus optimizes for this common case.

Morpheus optimizations are targeted primarily at minimizing per-layer costs. The main strategies are streamlining procedure linkage (since control is transferred between layers by procedure call) and factoring out computations that are repeated in multiple layers. In the best case, per-layer overhead can be reduced to two assembler jump instruction, one at the sender and one at the receiver.

Because Morpheus is intended to implement only the protocol subsystem of an operating system, the generated object code must interoperate with the operating system's object code. In particular, procedure calls in either direction between Morpheusgenerated machine code and "foreıgn" machine code adhere to the calling conventions of the foreign code.

Morpheus' optimizations cannot be duplicated by interprocedural optimization of a general purpose language. Morpheus optimizations are subject to two major constraints not usually encountered in general purpose optimization. First, it is not determined until runtime which protocol will be layered on top of which other protocol: it is unknown at compile time which callee procedure corresponds to a call site. Second, only the protocol subsystem is available for interprocedural optimization, not the entire operating system. Thus, protocols invoke and are invoked by foreign code, which has not been involved in the interprocedural optimizations. Even if these optimizations could be duplicated using general interprocedural optimization, it would involve considerable interprocedural analysis at compile time. Moreover, if separate compilation were to be supported, there would be additional compile time overhead to keep track of interprocedural dependences between separately compiled modules. Morpheus, which supports separate compilation, avoids these compile time penalties. In effect, the interprocedural analysis took place at language design time. 


\section{LittleEndian Header $\{$ unsigned seqNum; $\} \quad l^{*}$ declare header format * \\ Protocol $\{$ unsigned sendSeqNum; \} $\quad$ * declare Protocol state variables * \\ UnderSession \{ unsigned receiveSeqNum; \} $/^{*}$ declare UnderSession state variables *}

$f^{*}$ no programmer-declared state varlables needed for the other classes *

initProtocol ()$\{$ sendSeqNum $=1 ;\}$

initUnderSession ()$\{$ recelveSeqNum $=0 ;\}$

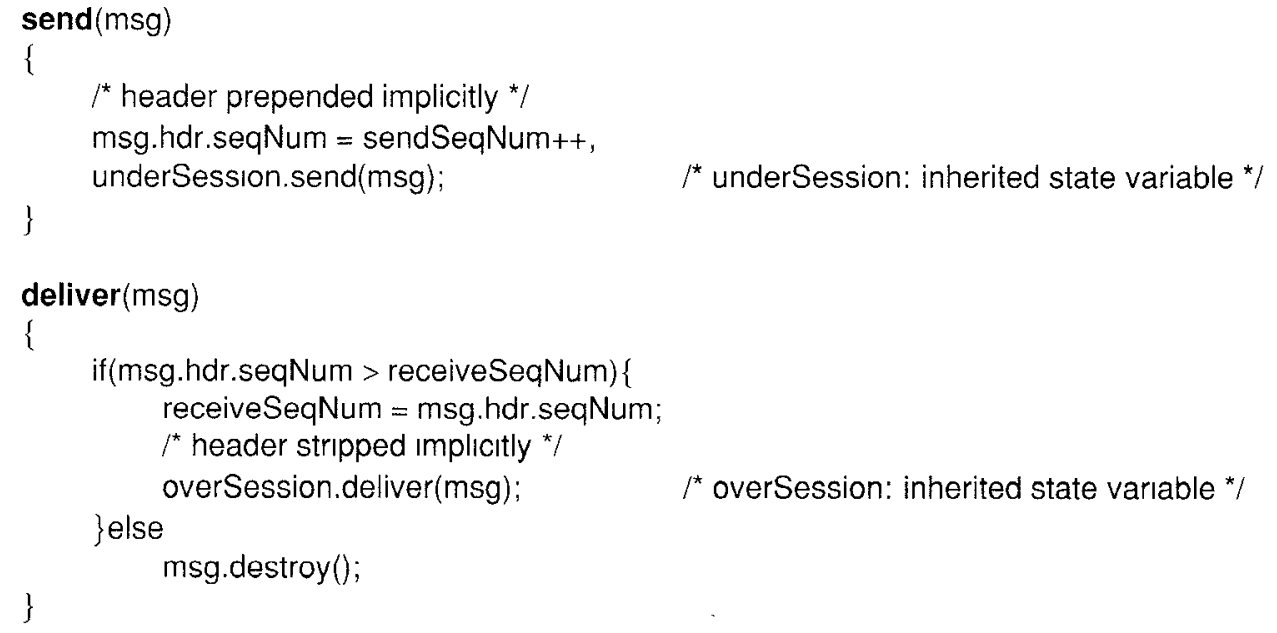

Figure 7: A worker protocol program

Before presenting the optımization techniques, we briefly review procedure call conventions for modern RISC architectures. ' The caller places the calling arguments in registers designated for that purpose. If there are many arguments, the excess arguments are passed via the stack. The caller then executes a jump-to-subroutine, which moves the return address into a designated register and transfers control to the callee. The callee then updates the stack pointer to leave enough space on the stack for local varıables, temporary varıables, callee saved registers, and arguments to be passed to procedures called by the callee. Any registers that need to be saved, including the return address register, are then saved on the stack. By convention, certain registers (callee save registers) must have their contents saved and restored by the callee if it uses them; certan other registers (culler save registers) may be used freely, but must be saved and restored around a call site by the caller if they are to hold a live value across the call. In preparation for returning, the callee puts the result in a designated register. It then restores any saved registers, including the return address register, restores the stack pointer, and jumps to the return address.

\footnotetext{
'We have decided to not consider register windows in this work. as we expect them to play a diminishing role in future machine architectures
}

\subsection{Specific Techniques}

We now identify five optimization techniques employed by the Morpheus compiler. For clarity, the techniques are described in terms of send; they apply equally to deliver.

\subsubsection{Dedicated Message Registers}

Consider send's message parameter, which fits in a register because it is implemented as a pointer. If send calls any procedures (other than those which take the message as an argument, in the same order in the argument list), the message has to be saved so that another argument can be passed in the argumentpassing registers. Ultimately it must be restored to its original argument-passing register to be passed to the next layer's send. Morpheus modifies the parameter passing convention by setting aside a register specifically to pass the message. This register is selected from among the callee save registers. This way it is efficiently accessible in a register, and what's more, that register need not be freed across subsequent calls to either the next layer's send or any other procedures.

The part of the message used most heavily is its header. A pointer to the message header is used to access or modify fields in the header, and is incremented or decremented to prepend or strip headers. Morpheus optımizes for this by designating 


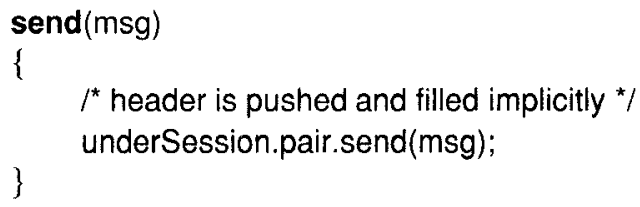

Figure 8: A multiplexor protocol program

a callee save register for passing the header pointer explicitly along with the message object of which it is a part. This eliminates memory accesses otherwise necessary to read or write the header pointer state variable in the message object, and does so using a register that need not be saved across calls.

Message and header registers are initialized when the message is created, either to be sent or because it was just received via a network device. Also, the original contents of the two registers used are saved at that same time, and restored upon return. This overhead is amortized over the number of layers in the send to obtain a per-layer cost. The message and header registers can potentially be reallocated within a send if registers are in sufficiently short supply or if a second message must be passed, but this case is the exception.

All these implementation details are concealed from the Morpheus programmer, who sees only operations on a Message object.

\subsubsection{Inline Substitution of Support Routines}

Morpheus provides built-in utilities for manipulating messages, mapping identifiers, and setting timers. Morpheus optimizes for their frequent use by substituting their code inline. The benefit of inlining is that procedure linkage code is eliminated and more context is exposed for conventional optimization. The costs of inline substitution are increased compile time and increased object code size. These costs are held to reasonable limits in Morpheus because the set of inlined procedures is fixed and small, and there is only one level of inlining-a procedure is never inlined into another procedure that is itself inlined.

\subsubsection{Eliminating Header Bounds Checking}

The most frequent utility operations are pushing (prepending) and popping (stripping) headers. Although pushing a header usually amounts to incrementing a pointer, it can involve considerable bounds checking even in the case where no bounds are exceeded. Morpheus optimizes this away by allocating sufficient header space to each message as it is created, thereby ensuring that the header will not overflow. This is possible because the runtime system can determine the largest combined header that can possibly to prepended to a message based on the headers declared by the protocols in the current protocol graph.

\subsubsection{Short-Circuit Return}

Most often. the last action taken in a send is to invoke the next layer's send. When the lower send returns, the original send is done and also returns. Morpheus short-circuits such returns in a manner similar to optimizations for tail recursion, so that sends with no further work are bypassed in the sequence of procedure returns. Before calling the lower send, the current send restores all registers including the stack pointer. It then jumps to the lower send, but instead of giving a return address in the current send, it gives the return address provided by the current send's caller.

This short-circuit return optimization in itself saves relatively little-a single jump assembler instruction per layer on the MIPS processor. However, it contributes to another, conventional optimization that is significant. If there are no procedure calls in a send operation, then that function can omit saving and restoring the return address register and updating and restoring the stack pointer. For this purpose, the short-circuit return effectively eliminates a procedure call. After applying short-circuiting and inline substitution, and eliminating bounds checking, a significant number of send operations qualify as having no procedure calls. This occurs frequently since the typical Morpheus protocol is relatively simple.

A variation on this optimization takes advantage of knowledge about the likelihood of executing various branches in the utility operations. Consider a send in which the sole procedure call is in some inlined utility code in a branch that is known to be infrequently taken. Instructions to manage the return address and stack pointer registers-i.e., a "lazy stack"-are inserted just in that infrequent branch, so that they are executed only if necessary.

\subsubsection{Procedure Cloning}

Send almost always accesses instance variables in its OverSession objects since these hold connection state information and other information such as the appropriate lower level OverSession object. It also frequently accesses instance variables of the Sap and Protocol objects to which the Session object belongs. Morpheus optimizes for this by generating a customized version of the send object code for each OverSession. At compile time, Morpheus generates a template for each protocol's send. When an OverSession object is created at runtime, a copy of the template is created and filled in-i.e. object code is modifiedusing the addresses of the Session, Sap, and Protocol objects and 
the values of those state variables that are known to be constant. Most inherited state varrables are known to be constant because they have to do with connecting layers together, e.g. the OverSap corresponding to a UnderSap, or the source and destination host addresses in a multiplexor OverSession. A user-declared state variable can be flagged as a constant by a keyword. Chains of indirect pointers through memory are collapsed; for example, the address of the next layer's send replaces a chain of pointers that leads to it through the current layer's UnderSession and the next layer's OverSession. This also eliminates the need to pass the OverSession object as a parameter.

The end result of the technique is that constants are hardwired into the code (the constants are different for each clone, hence they can't be hardwired into an uncloned procedure). This reduces the number of instructions executed for each clone. More importantly, it eliminates the memory accessesdisproportionately costly on a RISC machine - that would otherwise be necessary to read these constants.

This technique is a form of procedure cloning [3]. A procedure can be cloned to partition calls to it based on interprocedural constants information, or more generally, the solution to any forward interprocedural data-flow problem [7]. Instead of a single procedure that must satisfy all calls, each clone is specralized to more efficiently handle its subset of the calls. While conventional procedure cloning takes place entırely at compile time, in Morpheus the necessary information-the Session object for which the procedure is benng cloned-is not available until runtime. Thus Morpheus" technique could also be classified as runtime code generation. The Synthesis kernel [13] achieves exceptional performance using a similar technique.

Morpheus' cloning has time and space costs. There is the time cost, paid at runtime, of making a copy of the template and filling in the appropriate constants. While this does occur at runtime, it is part of communication channel creation-not in the time-critical send path. The space cost is an extra copy of the send code for each OverSession; that is, one for each communication channel currently provided by a protocol. There is already a space cost associated with each channel-a contextstate. In Morpheus this is the OverSession object. The corresponding send clone could be considered a part of that state. Note also that each clone uses less space than an uncloned version of a procedure because of the simplifications enabled by the cloning, and because some of the context-state is hardwired into the code. The increase in code space can be bounded by simply ceasing cloning once a code space threshold has been reached, as proposed in [7] This would require keeping one uncloned version of each send procedure to operate on any OverSessions that weren't allocated their own clones.

Increased object code size due to inlining (not cloning) seems to have little effect on caching and virtual memory. [4] found no obvious evidence of either thrashing or instruction cache overflow, and cited previous reports of similar results. While these studies involved inlıning, they suggest that increased object code size due to cloning would likewise be free of significant performance penalties.

\subsection{Experimental Results}

To study the Impact of Morpheus" optimizations, we handoptimized MIPS assembler code that was obtained from a prototype implementation of Morpheus' objects. The prototype was implemented in $\mathrm{C}$ and compiled using the GNU $\mathrm{C}$ compiler. We then performed two experiments to quantify the effect of Morpheus' optımization strategy: counting instructions and measuring end-to-end latency.

\subsubsection{Instruction Counts}

The effect of a given optımizatıon depends on both the particular procedure and the other optimizations present. Hence, we have selected a particular protocol to use as an example, and report the effects as each optımization is applied to it in turn. The protocol is Sequencer, which was presented in the previous section. We focus on Sequencer's send operation. When Sequencer's send is invoked. it pushes a header onto the message. The header is filled in with a sequence number obtained from a Protocol state variable, which is then incremented. The message is then passed to the next protocol's send.

The results of the optimizations are summarized in Table 2. The first row of the table refers to the original, unoptimized version of the code, which consists of 45 assembler instructions. The final, optimized version consists of seven instructions.

\begin{tabular}{|r|r|r|}
\hline CUMULATIVE & INSTRS & REMAINING \\
OPTIMIZATIONS & ELIMINATED & INSTRS \\
\hline ORIGINAL VERSION & - & 45 \\
INLINE UTILITIES & 7 & 38 \\
ELIM BOUNDS CHECK & 15 & 23 \\
DEDICATED REGS & 4 & 19 \\
CLONING & 7 & 12 \\
SHORT-CIRCUIT & 5 & 7 \\
\hline
\end{tabular}

Table 2: Instruction Counts

Inlining the push of the header reduces the common path by seven instructions-essentially the code for procedure linkage with the header push procedure. Eliminating header bounds checking eliminates an additional fifteen instructions. It also eliminates all conditional branches, so the common path is also the only path.

Dedicating registers for passing the message and its header eliminates an additional four instructions. This optimization generally gives a greater benefit in cases where there are procedure calls before callıng the next layer's send (Sequencer has no such intermediate calls after applying the preceding optimızations); intermediate calls prohibit the message from remaining in an argument-passing register because that register is also used to pass arguments at the intermediate calls.

Cloning send eliminates another seven instructions. Several pointer indirections are short-circuited, and one less parameter is passed to the next send (i.e., its OverSession). Cloning and dedicated registers also each owe some of thelr benefit in this case to reducing by one the number of callee save registers needed.

Short-circuiting the return from the subsequent send results in the elimination of five more instructions. Short-circuiting the 
return makes it unnecessary to save the return address, which in turn makes it unnecessary to allocate stack storage.

The fully optimized Sequencer send consists of seven instructions: one to increment the header pointer, five to do "the real work", and one to jump to the next layer. But not all assembler instructions are equal. Loads and stores can take much more than the single cycle used by other instructions, just how much time being determined by the current state of the cache. The gap between processor speed and memory speed can only be expected to widen in the future, making memory accesses an even more dominating factor in performance. The original, unoptimized version of Sequencer's send includes 12 loads and seven stores: the optimized version has one load and two stores. all in "the real work". This reduction in the number of loads and stores is roughly proportionate to the overall reduction in the number of instructions, a factor of about six.

\subsubsection{Timing Measurements}

We also compared the performance of an implementation of UDP in the $x$-kernel with an equivalent protocol stack in Morpheus. Because UDP cannot be implemented in Morpheus-it performs functions belonging to two different shapes-- the Morpheus equivalent consists of two protocols: a multiplexor performing first-come-first-serve multiplexing, and a worker that records in the message header the length of a sent message and trims each received message to the length recorded in its header. Omission of the checksumming function is discussed below.

The purpose of this experiment was to verify whether Morpheus' purported performance advantages would result in measurably high performance. The $x$-kernel was used as the standard for comparison because we could obtain timing measurements for the $x$-kernel's UDP on the same processor (Decstation $5000 / 200$ ), and because the $x$-kernel is known to support high performance protocol implementations [10]. UDP was used as the basis for comparison because, while farly simple, it qualIfies as a "real protocol." and because it has a clear Morpheus equivalent.

We measured the end-to-end latency of our two versions of UDP - the time it takes one message to be sent and received, independent of all other protocols. The measurement was taken by sending and receiving ten million, 1-word messages, and dividing the elapsed time by ten million. In this experiment, latency was independent of message size because the optional UDP checksum was not performed. neither system copies a message to pass it between layers, and messages were not actually transmitted over a network. The $x$-kernel implementation took 24.57 microseconds, while the Morpheus equivalent took only 1.48 microseconds, a factor of 16 difference.

Two qualifications apply to this result. First, there is the issue of the accuracy of microbenchmarks and their susceptibility to cache effects. In these experiments, all messages were transmitted over the same data stream with no intervening messages, with source and destination sharing the same processor, and no flushing of the cache. This should represent a best case performance, with very little data cache effect.

Second, the figure quoted for the $x$-kernel is not strictly latency but also includes the time to return control through the protocol graph on both the receiving and sending sides. This returning of control would normally occur either in parallel with message transmission. or after the message has been received. but took place serially in our experiment because source and destination shared the same processor. In this particular case, the additional time is relatıvely insignificant because it only involves three procedure returns. This was not a factor for the Morpheus time because Morpheus' short-circuit return optimization avolds the cost of returning for the layers being measured.

Despite these qualifications, the magnitude of the difference argues strongly for a Morpheus performance advantage. The difference is not attributable solely to Morpheus' optimizations. however; two other aspects of Morpheus also figure prominently.

First, even though UDP's checksum option was not used in the test, the $x$-kernel version still set the checksum field to zero on the sending side, and tested it for equality to zero on the receiving side. The Morpheus equivalent did not have this overhead. We argue that this is a legitımate advantage, attributable to the "many, simple protocols" approach used by Morpheus. In a protocol graph composed of many, simple protocols, the option of having a checksum is implemented by having two paths through the graph, one with the checksumming layer and one without it.

Second, accessing message headers is a far more elaborate process for the $x$-kernel than for Morpheus. Because compound data types such as $\mathrm{C}$ structures conform to alignment restrictions that may not be satisfied by the space allocated to a message header, $x$-kernel protocols read and write from temporary headers that are copied to and from messages by protocol-specific functions that account for potential alignment differences. Byte swapping, if necessary, is performed at the same time. Header manipulations in Morpheus are more efficient for two reasons. First, Morpheus ensures that header fields in messages satisfy its alignment restrictions. This is accomplished by padding a header internally so that individual fields are aligned with respect to the start of the header, and padding a header "externally" (i.e. on the wire) to maintain the invariant that each header starts on a word boundary. Second, any byte-swapping is performed by in-line code generated by the compiler for assignments that appear in the source language program. Hence, no function calls are required for either alignment or byte order; message headers may be read and written directly as if they were ordinary records, with any necessary byte swapping taking place invisibly and efficiently.

\subsection{Discussion}

There are two conclusions to draw from these experimental results. The first is that by using optimization techniques available to a special purpose language, a Morpheus compiler can generate very fast object code.

The second conclusion is that per-layer overhead in Morpheus is negligible. By "per-layer overhead" we mean the additional end-to-end latency of a protocol that is due to implementing it as a distinct protocol instead of incorporating it in another protocol. Sequencer's overhead is four instructions: two from send and two from deliver. Protocols more complex than Sequencer entail more overhead (because they need stacks and temporary registers and so on), but the overhead at each end is still less than a procedure call. 
An argument could be made that combining multiple functions in a single protocol layer still results in less overhead. It is true that the relative overhead-the ratio of overhead to "real work"-generally decreases as the functionality is squeezed into fewer layers. However, performance is not the sole issue. Combining functions in a single layer buys performance at the expense of modularity and its advantages in developing, verifying, implementing, and maintaining complex functionality. Morpheus not only supports modularity at the right level of granularity-indivisible protocol functions-but also offers benefits beyond conventional static modularity by supporting runtime composition of modules. All these benefits come at an end-to-end latency cost of less than two procedure calls per layer.

Latency of the protocol stack is not the only performance issue for network software; maximizing end-to-end throughput is also a pressing problem. The work described in this paper is just a part of a larger effort to address both latency and bandwidth in the context of a more powerful programming environment. Moreover, even though improving end-to-end throughput is generally considered to be the more critical issue in highspeed networking, minimizing latency is still an important goal. Considerable effort has been expended optimizing the latency of TCP/IP [2], obtaining both a significant performance benefit, and evidence bearing on the claim that TCP/IP latency need not be a performance bottleneck. The optimization techniques we introduce are not specific to any one protocol stack such as TCP/IP; hence we obtain both a significant performance benefit for protocols of any functionality, and evidence bearing on the claim that protocollatency in general need not be a performance bottleneck. This paper emphasizes per-layer latency in particular to support our thesis that highly layered architectures need not entail any significant latency penalty over architectures with few layers.

\section{Concluding Remarks}

Morpheus is a special-purpose programming language that facilitates the implementation of efficient communication protocols. In the context of implementing network software, our objective is to explore the design space that lies between implementing protocols by hand in the host operating system, and automatically generating network software from formal specifications. The key to Morpheus is that it constrains the protocol programmer. Morpheus' constraints enforce a good design discipline, relieve the programmer of many low-level design and implementation tasks, and admit optimizations for high performance. A powerful constraint unique to Morpheus is that of protocol shape.

\section{References}

[1] D. D. Clark. Modularity and efficiency in protocol implementation. Request for Comments 817. MIT Laboratory for Computer Science, Computer Systems and Communications Group, July 1982.
[2] D. D. Clark, V. Jacobson, J. Romkey, and H. Salwen. An analysis of TCP processing overhead. IEEE Communications Magazine, 27(6):23-29, June 1989.

[3] K. D. Cooper. Interprocedural Data Flow Analysis in a Programming Environment. PhD thesis, Rice University, April 1983.

[4] K. D. Cooper, M. W. Hall, and L. Torczon. An experiment with inline substitution. Softure-Practice and Experience, 21(6):581-601, June 91.

[5] O.-J. Dahl and K. Nygaard. Simula-an Algol-based simulation language. Communications of the ACM, 9(9):671678, Sept. 1966.

[6] D. C. Feldmeier. Multiplexing issues in communication system design. In Proceedings of the SIGCOMM '90 Symposium, 1990.

[7] M. W. Hall. Managing Interprocedural Optimization. PhD thesis, Rice University, April 1991.

[8] G. J. Holzmann. Design and Validation of Computer Protocols. Prentice Hall, Englewood Cliffs, NJ, 1991.

[9] B. L. Horn. An introduction to object oriented programming, inheritance and method combination. Technical Report CMU-CS-87-127, Computer Science Department, Carnegie Mellon University, Jan. 1988.

[10] N. C. Hutchinson and L. L. Peterson. The $x$-Kernel: An architecture for implementing network protocols. IEEE Transactions on Software Engineering, 17(1):64-76, Jan. 1991.

[11] G. Kane. MIPS RISC Architecture. Prentice Hall, Englewood Cliffs, NJ, 1988.

[12] S. W. O'Malley and L. L. Peterson. A dynamic network architecture. ACM Transactions on Computer Systems, 10(2), May 1992.

[13] C. Pu, H. Massalin, and J. Ioannidis. The Synthesis kernel. Computing Systems, 1(1):11-32, winter 1988.

[14] B. Stroustrup. The $C++$ Programming Language. Addison Wesley, Reading, MA, 1986.

[15] D. L. Tennenhouse. Layered multiplexing considered harmful. In Proceedings of the 1st International Workshop on High-Speed Networks, Nov. 1989.

[16] G. v. Bochmann. Usage of protocol development tools: The results of a survey. In Protocol Specification. Testing, and Verification, VII, 1987.

[17] R. W. Watson and S. A. Mamrak. Gaining efficiency in transport services by appropriate design and implementation choices. ACM Transactions on Computer Systems, 5(2):97-120, May 1987. 